\title{
Study of Photofield Emission in GaAs Using
}

\section{Kronig-Penney Model}

\author{
Leishangthem Nirmala Devi ${ }^{1}$, Benjamin Vanlalruata ${ }^{1}$, Ram Kumar Thapa ${ }^{1,2^{*}}$ and A. H. Reshak ${ }^{3,4}$ \\ 1. Condensed Matter Theory Research Group, Department of Physics, Mizoram University, 796004, India \\ 2. Condensed Matter Physics Research Centre, Butwal, Rupendehi, Nepal \\ 3. New Technologies - Research Centre, University of West Bohemia, Univerzitni 8, 30614 Pilsen, Czech Republic \\ 4. Center of Excellence Geopolymer and Green Technology, School of Material Engineering, University Malaysia \\ Perlis, 01007 Kangar, Perlis, Malaysia
}

\begin{abstract}
In this letter, we present the calculated results of photofield emission current (PFEC) in gallium arsenide (GaAs) by using Kronig-Penney potential model of Thapa and Kar [1]. It is found that PFEC decreases from higher value towards lower value exponentially in the case of GaAs. The results of plot of PFEC against initial state energy with respect to Fermi energy showed a maximum at $2.55 \mathrm{eV}$. Occurrence of these maxima is correlated with the plot of DOS (Density of state) of GaAs obtained by using DFT (Density functional theory).
\end{abstract}

Key words: Photofield emission current, photon energy, vector potential, Kronig-Penney model, density functional theory.

\section{Introduction}

In PFE (Photofield emission) a metal is illuminated by an incident laser radiation of photon energy less than the work function $\phi$ of the metal. Since the photoexcited electrons lies below the Fermi level and also below the vacuum level, a strong electric field ( $10^{11} \mathrm{~V} / \mathrm{m}$ ) when applied to the surface of the metal causes the photoexcited electrons to tunnel through the surface potential barrier into the vacuum region producing PFEC (Photofield emission current).

In this report, we are presenting the results of calculation of photofield emission current in gallium arsenide (GaAs) by using Kronig-Penney potential model [1]. Here we are trying to correlate with density of state calculations by suing the density functional theory.

\section{Theory}

PFEC is calculated by using the formula [2]

\footnotetext{
*Corresponding author: Ram Kumar Thapa, professor,
} research fields: condensed matter physics.

$$
\frac{d j}{d E}=-\frac{e^{3}}{2 \hbar^{4} \omega^{3}} \frac{n}{\Omega}(\hat{\varepsilon} \cdot \hat{z})^{2} f(E-\hbar \omega) \int_{-V_{o}+\hbar \omega}^{E} d W \frac{D(W)\left|M_{f i}\right|^{2}}{[W(W-\hbar \omega)]^{\frac{1}{2}}}
$$

The matrix element $M_{f i}$ in Eq. (1) when expanded in one-dimension along $z$-axis is given by:

$$
\begin{aligned}
M_{f i}= & \int_{-d}^{0} \psi_{f}^{*} A_{z} \frac{d V}{d z} \psi_{i} d z+\int_{-d}^{0} \psi_{f}^{*} \frac{d^{2} A_{z}}{d z^{2}}\left(-i \hbar \frac{d}{d z}\right) \psi_{i} d z \\
& +\int_{-d}^{0} \psi_{f}^{*} \frac{d A_{z}}{d z}\left(-\hbar^{2} \frac{d^{2}}{d z^{2}}\right) \psi_{i} d z+\int_{-d}^{0} \psi_{f}^{*} \frac{d A_{z}}{d z} \psi_{i} d z
\end{aligned}
$$

The initial state wavefunction in matrix element $M_{f i}$ in Eq. (2) above had been calculated by Thapa and Kar [1] using the Kronig-Penney potential model for the solid by wavefunction matching at the surface defined by $\mathrm{z}=0$ plane. This is given by:

$$
\psi_{i}(z)= \begin{cases}\left(1-i P e^{-i \delta} \sin \delta\right) e^{i k_{i} z} & \\ -\left(P-i e^{i \delta} \sin \delta\right) e^{-i k_{i} z} & \\ T e^{-\chi z}, & z<0\end{cases}
$$

where, $\cot \delta=-\frac{k_{i}}{g}, \mathrm{~g}$ is the strength of the potential. 
Here final state wavefunctions and other parameters used are described by Thapa and Das [3] in detail. FORTRAN program is written to calculate PFEC by writing.

\section{Results and Discussion}

We discuss here the theoretical results of PFEC in the case of GaAs obtained by using the Kronig-Penney potential model. PFEC is calculated as a function of initial state energy $\left(E_{i}\right)$, photon energy $(\hbar \omega)$ and the applied high static electric field $(F)$. We have used the following theoretical input parameters for calculations of PFEC: Surface width $(d)=5.7363$ $\AA$, initial state energy $\left(E_{i}\right)=4.49888 \mathrm{eV}$, potential barrier height $\left(V_{0}\right)=10.2562 \mathrm{eV}$, work function $(\phi)=$ $3.5 \mathrm{eV}$, Fermi energy $\left(E_{F}\right)=5.5662 \mathrm{eV}$, scattering factor $(\alpha)=0.35$ and phase shift $(\delta)=-0.6416$.

In Fig. 1, we have plotted the calculated PFEC as a function of the applied electric field $(F)$ for three difference values of photon energies $\hbar \omega=3.22455$ $\mathrm{eV}, 3.85041 \mathrm{eV}$ and $4.20416 \mathrm{eV}$ respectively. We have chosen the initial state energy $E_{i}=1 \mathrm{eV}$ below Fermi level $\left(E_{F}=0.0\right)$. From the plot, we find that the value of applied electric field is increases PFEC decreases from a high value towards minimum in an exponential manner for all the three different values of photon energies. The exponential decrease in photofield emission current is due to presence of exponential term in calculation of transition probability $D(W)$ given by Eq. (9). From the plot, we also find that a higher value of PFEC for low photon energy value $\hbar \omega=3.22455 \mathrm{eV}$ whereas lower value of PFEC for large photon energy $\hbar \omega=4.20416 \mathrm{eV}$. This is due to the reason that PFEC given by Eq. (1) is inversely proportional to frequency of incident photon radiation. However, the variation of PFEC in all the cases of three different values of photon energies are similar in nature.

In Fig. 2, we have plotted the results of the calculated PFEC as a function of initial state energy $\left(E_{i}\right)$ for three different values of photon energies $\hbar \omega=$
3.22455, 3.85041 and $4.20416 \mathrm{eV}$ respectively. Here the value of applied electric field $\left(F_{a}\right)$ is equal to $1.6 \times$ $10^{11} \mathrm{~V} / \mathrm{m}$. and initial state energy $E_{i}$ is chosen below the Fermi level $\left(E_{F}=0.0\right)$. From the plot, we have seen that PFEC for photon energy $\hbar \omega=3.22455 \mathrm{eV}$ occurs a maximum peak at $E_{i}=2.55 \mathrm{eV}$ below the Fermi level. With the further decrease in initial state energy PFEC decreases but becomes minimum at about $E_{i}=2.75 \mathrm{eV}$ below the Fermi level. We have also plotted PFEC for photon energies $\hbar \omega=3.85041$ $\mathrm{eV}$ and $4.20416 \mathrm{eV}$ respectively. From this same plot, we also see that maximum peaks in PFEC occur at same initial state energy at $E_{i}=2.55 \mathrm{eV}$ for both photon energies $3.85041 \mathrm{eV}$ and $4.20416 \mathrm{eV}$ below Fermi level. Here the peak in PFEC is higher for photon energy $\hbar \omega=3.22455 \mathrm{eV}$ than for photon energies $3.85041 \mathrm{eV}$ and $4.20416 \mathrm{eV}$. Therefore, the

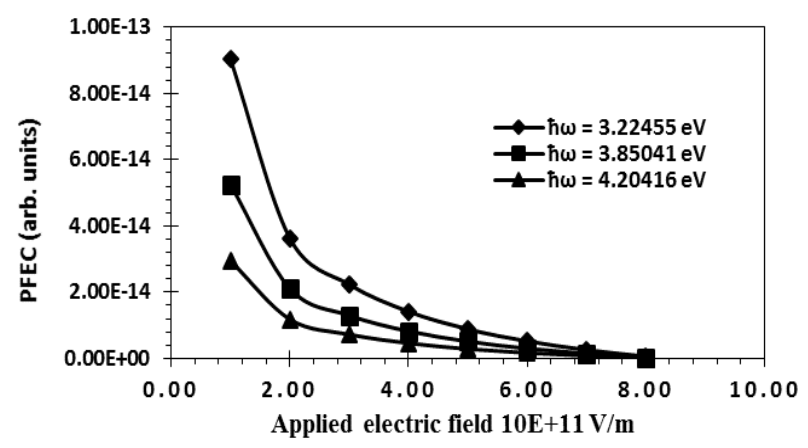

Fig. 1 Plot of PFEC against applied electric field for three values of photon energies $\hbar \omega=3.22455,3.85041$ and $4.20416 \mathrm{eV}$.

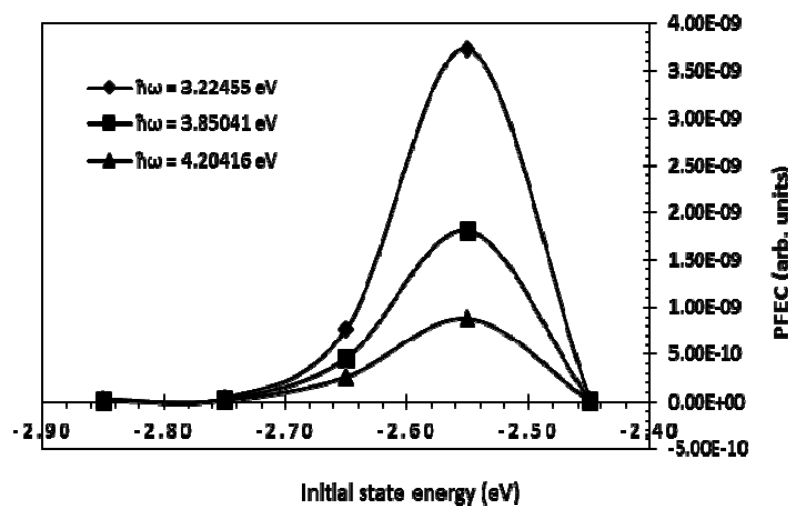

Fig. 2 Plot of PFEC against initial state energy for three values of photon energies $\hbar \omega=3.22455,3.85041$ and $4.20416 \mathrm{eV}$. Here applied static electric field is $1.6 \times 10^{11}$ $\mathrm{V} / \mathrm{m}$ and Fermi level $\boldsymbol{E}_{\boldsymbol{F}}=\mathbf{0 . 0}$ is taken as reference level. 


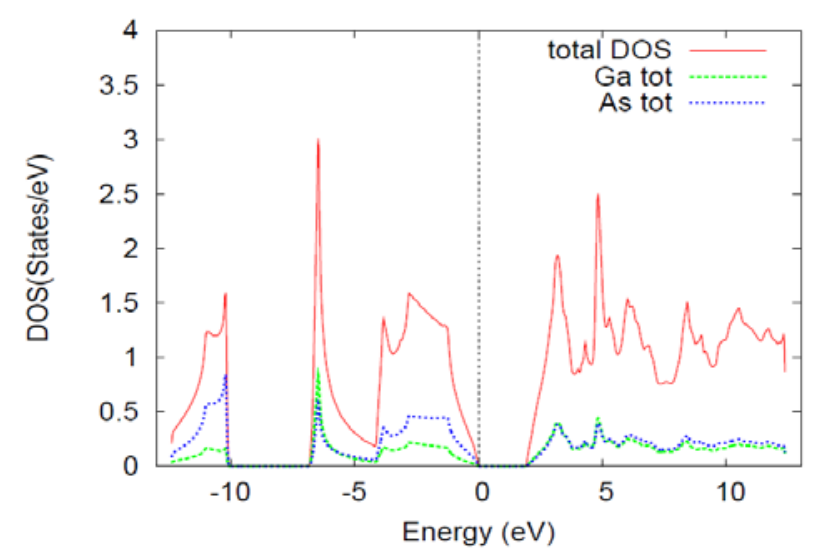

Fig. 3 Plot of total DOS and partial DOS of Ga and As.

higher value of PFEC for low photon energy value $\hbar \omega=3.22455 \mathrm{eV}$ whereas low value of PFEC for high photon energy $\hbar \omega=4.20416 \mathrm{eV}$. This is due to that PFEC is inversely proportional to frequency of incident photon radiation.

The origin of peaks at $2.56 \mathrm{eV}$ below the Fermi level as shown in Fig. 2 can be correlated with the occurrence of peak at $2.77 \mathrm{eV}$ in the DOS plots as shown in Fig. 3. This means that the origin of peaks at $2.56 \mathrm{eV}$ below the Fermi level as shown in Fig. 2 is due to maximum contribution by $s$ and $p$ states of Ga and $p$ state of As.

\section{Conclusions}

In calculating PFEC we have used the Kronig-Penney potential model which have been used by Thapa and N. Kar [1]. It is found that the behavior of PFEC as a function of applied field and initial state energy shows similar trends also in the case of GaAs. The occurrence of peak in PFEC at initial state $E_{i}=$ $2.55 \mathrm{eV}$ below the Fermi energy can be addressed due to band structure effects. This is evident from the plots of density of state (DOS) in GaAs which is shown in Fig. 3. However the discrepancy in the location of peaks as shown in Figs. 2 and 3 can be attributed to the choice of the wavefunctions to evaluate the matrix element. However, few drawbacks are still existing which must be attended for better accuracy. For example, we have used the real and imaginary dielectric constants which had been calculated by using the density functional theory. This was included to the model as proposed by Bagchi and Kar [6]. We are trying to correlate appropriately the dielectric model in the context of density function theory to calculate correctly vector potentials for transition probability.

\section{Acknowledgments}

I am grateful to Mizoram University UGC fellowship, RKT acknowledges a grant from SERB (DST, India) via grant No. EMR/2015/001407, Dt.11 March 2016. A. H. Reshak would like to acknowledges the CENTEM project, Reg. No. CZ.1.05/2.1.00/03.0088, co-funded by the ERDF, as part of the Ministry of Education, Youth and Sports OP RDI program and, in the follow-up sustainability stage, supported through CENTEM PLUS (LO1402) by financial means from the Ministry of Education, Youth and Sports under the "National Sustainability Program I. Also would like to acknowledge the MetaCentrum (LM2010005) and CERIT-SC (CZ.1.05/3.2.00/08.0144) infrastructures.

\section{References}

[1] Thapa, R. K., and Kar, N. 1988. "Photoemission Calculation from Band States Using Krong-Penney Model \& Spatially Varying Photon Field.” Indian J. pure appl. Phys 26: 620-3.

[2] Gao, Y. 1986. "Photofield Emission Study of Metal Surfaces.” Ph.D. thesis, Purdue University, Purdue.

[3] Thapa, R. K. and Das, G. 2005. "A Simple Theory of Photofield Emission from the Surface of a Metal.” Intl. Jour Mod. Phys. B19: 3141-9.

[4] Thapa, R. K., Das, G., Gurung, S. R., Sharma, B. I., Patra, P. K. and Ghimire, M. P. 2007. "Photofield Emission Calculation by Using Projector Operator Method.” Mod. Phys Letts. B21: 501-1507.

[5] Bagchi, A., and Kar, N. 1978. "Reflection Effects in Angle-Resolved Photo Emission from Surface States on Metal.” Phys. Rev. B18: 5240-7. 